\title{
Coordination of a neutral ligand to a metal center of oxohalido anions: fact or fiction?
}

\section{Supporting information}

Anton Kokalj, ${ }^{\text {acc }}$ Žiga Zupanek, ${ }^{\text {b,c }}$ Melita Tramšek ${ }^{\mathrm{b}}$ and Gašper Tavčar ${ }^{\mathrm{b}, c *}$

${ }^{a}$ Department of Physical and Organic Chemistry, Jožef Stefan Institute, Jamova 39, 1000 Ljubljana, Slovenia

${ }^{\mathrm{b}}$ Department of Inorganic Chemistry and Technology, Jožef Stefan Institute, Jamova 39, 1000 Ljubljana, Slovenia

c Jožef Stefan International Postgraduate School, Jamova 39, 1000 Ljubljana, Slovenia

\section{Table of content}

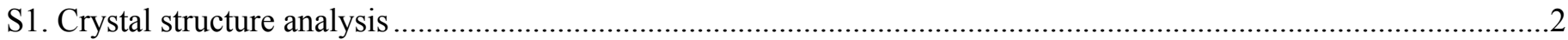

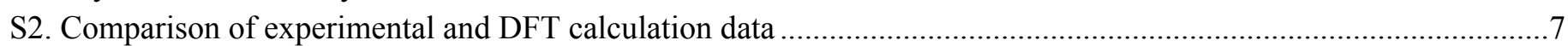

S3. MOPDOS of the $\left[\mathrm{VOF}_{4}(\mathrm{THF})\right]^{-}$and $\left[\mathrm{VOF}_{4}(\mathrm{Py})\right]^{-}$complexes: projections to the $\mathrm{VOF}_{4}{ }^{-}$anion ..............................

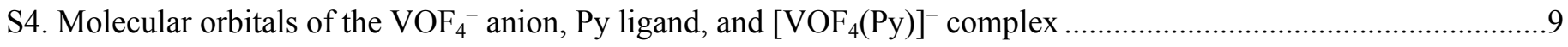

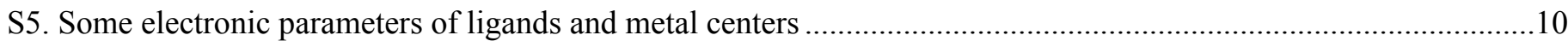

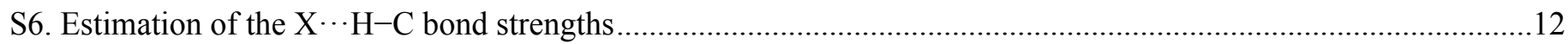

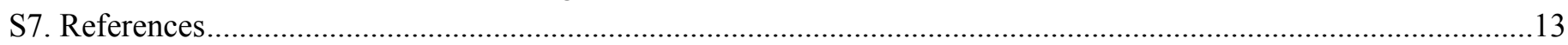




\section{S1. Crystal structure analysis}

Table S1. Summary of crystal data and structure refinement for $\left[\left(\mathrm{L}^{\mathrm{Dipp}}\right) \mathrm{H}\right]\left[\mathrm{VOF}_{4}(\mathrm{THF})\right](\mathbf{1})$ and $\left[\left(\mathrm{L}^{\mathrm{Dipp}}\right) \mathrm{H}\right]\left[\mathrm{VOF}_{4}(\mathrm{Py})\right](\mathbf{2})$.

\begin{tabular}{|c|c|c|}
\hline Compound & {$\left[\left(\mathrm{L}^{\mathrm{Dipp}}\right) \mathrm{H}\right]\left[\mathrm{VOF}_{4}(\mathrm{THF})\right](\mathbf{1})$} & {$\left[\left(\mathrm{L}^{\mathrm{Dipp}}\right) \mathrm{H}\right]\left[\mathrm{VOF}_{4}(\mathrm{Py})\right](\mathbf{2})$} \\
\hline CCDC No. & 2065280 & 2065279 \\
\hline Formula & $\mathrm{C}_{27} \mathrm{H}_{37} \mathrm{~N}_{2} \cdot \mathrm{C}_{4} \mathrm{H}_{8} \mathrm{~F}_{4} \mathrm{O}_{2} \mathrm{~V}$ & $2\left(\mathrm{C}_{27} \mathrm{H}_{37} \mathrm{~N}_{2}\right) \cdot 2\left(\mathrm{C}_{5} \mathrm{H}_{5} \mathrm{~F}_{5} \mathrm{NOV}\right)$ \\
\hline$F_{\mathrm{w}}$ & 604.63 & 1223.25 \\
\hline$T[\mathrm{~K}]$ & 150 & 150 \\
\hline Crystal system & Monoclinic & Triclinic \\
\hline Space group & $P 2_{1} / c$ & $P-1$ \\
\hline$a[\AA]$ & $12.6595(1)$ & $12.5745(3)$ \\
\hline$b[\AA]$ & $12.7750(1)$ & $12.7175(3)$ \\
\hline$c[\AA]$ & $19.6070(2)$ & $20.5561(5)$ \\
\hline$\alpha\left[^{\circ}\right]$ & 90 & $88.902(2)$ \\
\hline$\beta\left[^{\circ}\right]$ & $93.396(1)$ & $81.187(2)$ \\
\hline$\gamma\left[{ }^{\circ}\right]$ & 90 & $87.142(2)$ \\
\hline$V\left[\AA^{3}\right]$ & $3165.38(5)$ & $3244.2(1)$ \\
\hline$Z$ & 2 & 2 \\
\hline$\rho_{\text {calc }}\left[\mathrm{g} / \mathrm{cm}^{3}\right]$ & 1.269 & 1.252 \\
\hline Crystal size $[\mathrm{mm}]$ & $0.49 \times 0.35 \times 0.27$ & $0.68 \times 0.29 \times 0.17$ \\
\hline Radiation type & $\mathrm{Cu} \mathrm{K} \alpha$ & $\mathrm{Cu} \mathrm{K} \alpha$ \\
\hline$\lambda[\AA]$ & 1.54184 & 1.54184 \\
\hline$\mu\left[\mathrm{mm}^{-1}\right]$ & 3.066 & 2.986 \\
\hline$F(000)$ & 1280 & 1288 \\
\hline \multirow[t]{2}{*}{$\Theta_{\max }\left[{ }^{\circ}\right]$} & 67.080 & 67.073 \\
\hline & $-15 \leq h \leq 15$ & $-14 \leq h \leq 15$ \\
\hline \multirow[t]{2}{*}{ Index ranges } & $-15 \leq k \leq 15$ & $-15 \leq k \leq 15$ \\
\hline & $-23 \leq l \leq 23$ & $-24 \leq l \leq 24$ \\
\hline Reflections collected & 71450 & 34582 \\
\hline Independent reflections & 5637 & 11479 \\
\hline Reflections with $[I>2 \sigma(I)]$ & 5262 & 10344 \\
\hline$R_{\text {int }}$ & 0.0330 & 0.0228 \\
\hline$R_{\text {sigma }}$ & 0.0117 & 0.0220 \\
\hline Data/restrains/parameters & $5637 / 13 / 415$ & $11479 / 0 / 755$ \\
\hline$S^{[\mathrm{a}]}$ & 1.090 & 1.032 \\
\hline$R_{1}{ }^{[\mathrm{b}]}, w R_{2}{ }^{[\mathrm{cc}]}[I>2 \sigma(I)]$ & $0.0376,0.1004$ & $0.0398,0.1087$ \\
\hline$R_{1}{ }^{[\mathrm{b}]}, w R_{2}^{[\mathrm{c}]}[$ all data $]$ & $0.0400,0.1019$ & $0.0449,0.1131$ \\
\hline$\Delta \rho_{\min }, \Delta \rho_{\max }\left[\mathrm{e} \AA^{-3}\right]$ & $-0.356,0.412$ & $-0.397,0.684$ \\
\hline
\end{tabular}

[a] $S=\left[\Sigma\left(w\left(F_{\mathrm{o}}^{2}-F_{\mathrm{c}}^{2}\right)^{2}\right) /\left(N_{\mathrm{o}}-N_{\mathrm{p}}\right)\right]^{1 / 2}$.

${ }^{[b]} R_{1}=\| F_{\mathrm{o}}|-| F_{\mathrm{c}}|| \Sigma\left|F_{\mathrm{o}}\right|$.

[c] $w R_{2}=\left[\Sigma\left(w\left(F_{\mathrm{o}}^{2}-F_{\mathrm{c}}^{2}\right)^{2}\right) / \Sigma\left(w\left(F_{\mathrm{o}}^{2}\right)^{2}\right)\right]^{1 / 2}$. 
Table S2. Bond lengths $[\AA]$ and angles $\left[{ }^{\circ}\right]$ for both domains of the $\left[\left(\mathrm{L}^{\mathrm{Dipp}}\right) \mathrm{H}\right]\left[\mathrm{VOF}_{4}(\mathrm{THF})\right](\mathbf{1})$ as well as the occupancy for domain A and B.

\begin{tabular}{|c|c|c|}
\hline Domain of THF & Occupancy & \\
\hline$\overline{\mathrm{A}}$ & $46.1(9) \%$ & \\
\hline B & $53.9(9) \%$ & \\
\hline Intramolecular bond & Bond length $[\AA]$ & Bond valence $^{[\mathrm{a}]}$ \\
\hline $\mathrm{V}=\mathrm{O}$ & $1.561(2)$ & 1.923 \\
\hline $\mathrm{V}-\mathrm{O}_{\mathrm{THF}}$ & $\begin{array}{l}\text { 2.403(7) }(\mathrm{O} 30 \mathrm{~A} ; \text { domain } \mathrm{A}) \\
\text { 2.464(6) (O30B; domain } \mathrm{B})\end{array}$ & $\begin{array}{l}0.198(\mathrm{O} 30 \mathrm{~A}) \\
0.168(\mathrm{O} 30 \mathrm{~B})\end{array}$ \\
\hline \multirow{5}{*}{$\mathrm{V}-\mathrm{F}$} & $1.819(1)(\mathrm{F} 1)$ & 0.745 \\
\hline & $1.805(1)(\mathrm{F} 2)$ & 0.774 \\
\hline & $1.806(1)(\mathrm{F} 3)$ & 0.771 \\
\hline & $1.821(1)(\mathrm{F} 4)$ & 0.741 \\
\hline & $\Sigma s_{\mathrm{V}}$ & $\begin{array}{l}5.152(\text { domain } A) \\
5.122(\text { domain } B)\end{array}$ \\
\hline Intermolecular bond & Bond length $[\AA]$ & \\
\hline $\mathrm{F}-\mathrm{C} 2$ & $\begin{array}{l}3.004(2)(\mathrm{F} 1) \\
2.986(2)(\mathrm{F} 4)\end{array}$ & \\
\hline $\mathrm{F}-\mathrm{C} 4$ & $\begin{array}{l}3.936(2)(\mathrm{F} 2) \\
2.946(2)(\mathrm{F} 3)\end{array}$ & \\
\hline $\mathrm{F}-\mathrm{C} 5$ & $\begin{array}{l}3.081(2)(\mathrm{F} 2) \\
3.065(2)(\mathrm{F} 3)\end{array}$ & \\
\hline Intramolecular angle & Angle $\left[^{\circ}\right]$ & \\
\hline $\mathrm{O}=\mathrm{V}-\mathrm{F}$ & $\begin{array}{l}99.64(7)(\mathrm{F} 1) \\
100.21(9)(\mathrm{F} 2) \\
100.53(7)(\mathrm{F} 3) \\
98.69(8)(\mathrm{F} 4)\end{array}$ & \\
\hline $\mathrm{O}_{\mathrm{THF}}-\mathrm{V}-\mathrm{F}$ & $\begin{array}{l}\text { 82.1(2) }(\mathrm{F} 1, \mathrm{O} 30 \mathrm{~A}, \text { domain } \mathrm{A}) \\
\text { 88.3(2) }(\mathrm{F} 2, \mathrm{O} 30 \mathrm{~A}, \text { domain } \mathrm{A}) \\
77.7(2)(\mathrm{F} 3, \mathrm{O} 30 \mathrm{~A}, \text { domain } \mathrm{A}) \\
72.9(2)(\mathrm{F} 4, \mathrm{O} 30 \mathrm{~A}, \text { domain } \mathrm{A}) \\
76.3(2)(\mathrm{F} 1, \mathrm{O} 30 \mathrm{~B}, \text { domain } \mathrm{B}) \\
77.1(2)(\mathrm{F} 2, \mathrm{O} 30 \mathrm{~B}, \text { domain } \mathrm{B}) \\
83.6(2)(\mathrm{F} 3, \mathrm{O} 30 \mathrm{~B}, \text { domain } \mathrm{B}) \\
83.9(2)(\mathrm{F} 4, \mathrm{O} 30 \mathrm{~B}, \text { domain } \mathrm{B})\end{array}$ & \\
\hline $\mathrm{O}=\mathrm{V}-\mathrm{O}_{\mathrm{THF}}$ & $\begin{array}{l}\text { 171.3(2) }(\mathrm{O} 30 \mathrm{~A}, \text { domain } \mathrm{A}) \\
175.1(2)(\mathrm{O} 0 \mathrm{~B}, \text { domain } \mathrm{B})\end{array}$ & \\
\hline $\mathrm{F}-\mathrm{V}-\mathrm{F}_{\text {cis }}$ & $\begin{array}{l}88.89(6)(\mathrm{F} 1, \mathrm{~F} 2) \\
86.60(5)(\mathrm{F} 1, \mathrm{~F} 4) \\
88.95(6)(\mathrm{F} 2, \mathrm{~F} 3) \\
88.96(5)(\mathrm{F} 3, \mathrm{~F} 4)\end{array}$ & \\
\hline $\mathrm{F}-\mathrm{V}-\mathrm{F}_{\text {trans }}$ & $\begin{array}{l}159.78(6)(\mathrm{F} 1, \mathrm{~F} 3) \\
161.05(7)(\mathrm{F} 2, \mathrm{~F} 4)\end{array}$ & \\
\hline Intermolecular angle & Angle $\left[{ }^{\circ}\right]$ & \\
\hline Plane $_{\text {equatorial-F}}-$ Plane $_{\text {imidazolium }}$ & $\begin{array}{l}\text { 87.0(5) }(\mathrm{C} 2 \text { toward anion }) \\
23.9(6)(\mathrm{C} 4, \mathrm{C} 5 \text { toward anion })\end{array}$ & \\
\hline
\end{tabular}


Table S3. Bond lengths $[\AA]$ and angles $\left[{ }^{\circ}\right]$ for both subunits of the $\left[\left(\mathrm{L}^{\mathrm{Dipp}}\right) \mathrm{H}\right]\left[\mathrm{VOF}_{4}(\mathrm{Py})\right](\mathbf{2})$.

\begin{tabular}{|c|c|c|c|c|}
\hline \multirow[b]{2}{*}{ Intramolecular bond } & \multicolumn{2}{|l|}{ Subunit 1} & \multicolumn{2}{|l|}{ Subunit 2} \\
\hline & Bond length $[\AA]$ & Bond valence ${ }^{[\mathrm{a}]}$ & Bond length $[\AA]$ & Bond valence ${ }^{[\mathrm{a}]}$ \\
\hline $\mathrm{V}=\mathrm{O}$ & $1.570(2)$ & 1.877 & $1.574(2)$ & 1.857 \\
\hline $\mathrm{V}-\mathrm{N}_{\mathrm{Py}}$ & $2.399(2)$ & 0.233 & $2.406(2)$ & 0.229 \\
\hline \multirow{5}{*}{$\mathrm{V}-\mathrm{F}$} & $1.840(1)(\mathrm{F} 1)$ & 0.704 & $1.832(1)(\mathrm{F} 5)$ & 0.719 \\
\hline & $1.804(1)(\mathrm{F} 2)$ & 0.776 & $1.805(1)(\mathrm{F} 6)$ & 0.774 \\
\hline & $1.831(1)(\mathrm{F} 3)$ & 0.721 & $1.821(1)(\mathrm{F} 7)$ & 0.741 \\
\hline & $1.811(1)(\mathrm{F} 4)$ & 0.761 & $1.815(1)(\mathrm{F} 8)$ & 0.753 \\
\hline & $\Sigma s_{\mathrm{V}}$ & 5.072 & $\Sigma s_{\mathrm{V}}$ & 5.072 \\
\hline Intermolecular bond & \multicolumn{2}{|l|}{ Bond length $[\AA]$} & \multicolumn{2}{|l|}{ Bond length $[\AA]$} \\
\hline \multirow{6}{*}{$\mathrm{F}-\mathrm{C}_{\text {imidazolium }}$} & \multicolumn{2}{|l|}{$2.896(2)(\mathrm{F} 1, \mathrm{C} 2)$} & \multicolumn{2}{|l|}{$2.888(2)(\mathrm{F} 5, \mathrm{C} 31)$} \\
\hline & \multicolumn{2}{|l|}{$4.175(2)(\mathrm{F} 2, \mathrm{C} 2)$} & \multicolumn{2}{|l|}{$4.251(3)(\mathrm{F} 6, \mathrm{C} 31)$} \\
\hline & \multicolumn{2}{|c|}{$4.032(2)(\mathrm{F} 3, \mathrm{C} 33)$} & \multicolumn{2}{|l|}{$3.169(2)(\mathrm{F} 7, \mathrm{C} 4)$} \\
\hline & \multicolumn{2}{|c|}{$3.100(2)(\mathrm{F} 3, \mathrm{C} 34)$} & \multicolumn{2}{|l|}{$4.051(2)(F 7, C 5)$} \\
\hline & \multicolumn{2}{|c|}{ 2.921(2) (F4, C33) } & \multicolumn{2}{|l|}{$3.022(2)(\mathrm{F} 8, \mathrm{C} 4)$} \\
\hline & \multicolumn{2}{|c|}{$2.877(2)(\mathrm{F} 4, \mathrm{C} 34)$} & \multicolumn{2}{|l|}{$2.984(2)(\mathrm{F} 8, \mathrm{C} 5)$} \\
\hline Intramolecular angle & \multicolumn{2}{|l|}{ Angle $\left[^{\circ}\right]$} & \multicolumn{2}{|l|}{ Angle $\left[{ }^{\circ}\right]$} \\
\hline \multirow{4}{*}{$\mathrm{O}=\mathrm{V}-\mathrm{F}$} & \multicolumn{2}{|l|}{$99.45(9)(\mathrm{F} 1)$} & \multicolumn{2}{|l|}{$98.62(9)(\mathrm{F} 5)$} \\
\hline & \multicolumn{2}{|l|}{$99.49(8)(\mathrm{F} 2)$} & \multicolumn{2}{|l|}{$98.07(8)(\mathrm{F} 6)$} \\
\hline & \multicolumn{2}{|l|}{$99.13(9)(\mathrm{F} 3)$} & $99.34(9)(\mathrm{F} 7)$ & \\
\hline & $98.61(9)(\mathrm{F} 4)$ & & $100.40(7)(F 8)$ & \\
\hline & $80.70(6)(\mathrm{F} 1)$ & & $79.66(6)(\mathrm{F} 5)$ & \\
\hline & $80.14(6)(\mathrm{F} 2)$ & & $79.25(6)(\mathrm{F} 6)$ & \\
\hline $\mathrm{N}_{\mathrm{Py}}-\mathrm{V}-\mathrm{F}$ & $80.73(6)(\mathrm{F} 3)$ & & $82.53(6)(\mathrm{F} 7)$ & \\
\hline & $81.75(6)(\mathrm{F} 4)$ & & $82.28(5)(\mathrm{F} 8)$ & \\
\hline $\mathrm{O}=\mathrm{V}-\mathrm{N}_{\mathrm{Py}}$ & 179.61(9) & & $176.78(8)$ & \\
\hline & $89.34(6)(\mathrm{F} 1, \mathrm{~F} 2)$ & & $89.54(7)(\mathrm{F} 5, \mathrm{~F} 6)$ & \\
\hline $\mathrm{F}-\mathrm{V}-\mathrm{F}$ & $86.75(5)(F 1, F 4)$ & & $87.53(6)(\mathrm{F} 5, \mathrm{~F} 8)$ & \\
\hline$\Gamma-V-\Gamma_{\text {cis }}$ & $89.00(6)(\mathrm{F} 2, \mathrm{~F} 3)$ & & $90.52(7)(\mathrm{F} 6, \mathrm{~F} 7)$ & \\
\hline & $89.08(5)(\mathrm{F} 3, \mathrm{~F} 4)$ & & $86.69(6)(\mathrm{F} 7, \mathrm{~F} 8)$ & \\
\hline & $161.37(6)(\mathrm{F} 1, \mathrm{~F} 3$ & & $161.85(7)(\mathrm{F} 5, \mathrm{~F} 7)$ & \\
\hline $\mathrm{F}-\mathrm{V}-\mathrm{F}_{\text {trans }}[\mathrm{l}]$ & $161.87(7)(\mathrm{F} 2, \mathrm{~F} 4$ & & $161.53(6)(\mathrm{F} 6, \mathrm{~F} 8)$ & \\
\hline Intramolecular angle & Angle $\left[{ }^{\circ}\right]$ & & Angle $\left[{ }^{\circ}\right]$ & \\
\hline Plane $_{\text {equatorial } F}-$ Plane $_{\text {equatorial } F}$ & $80.80(6)$ (subunit & ubunit 2) & & \\
\hline Plane $_{\text {equatorial F}}-$ Plane $_{\text {Py }}$ & $83.82(8)$ & & $88.8(1)$ & \\
\hline & 42.04(8) (F1-O1- & & $56.8(3)(\mathrm{F} 5-\mathrm{O} 2-\mathrm{F} 7$ & \\
\hline Plane $_{\mathrm{FOF}}-$ Plane $\mathrm{Py}_{\mathrm{P}}$ & $47.90(7)(\mathrm{F} 2-\mathrm{O} 1-$ & & 32.9(3) (F6-O2-F8 & \\
\hline Plane $_{\text {equatorial } \mathrm{F}}-$ Plane $_{\text {imidazolium }}$ & $70.31(5)(\mathrm{C} 2$ tow & anion) & $76.00(8)(\mathrm{C} 31$ tow & anion) \\
\hline 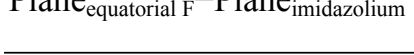 & $24.14(7)(\mathrm{C} 33, \mathrm{C}$ & ward anion) & $20.6(1)(\mathrm{C} 4, \mathrm{C} 5$ to & rd anion) \\
\hline
\end{tabular}

[a] Bond valence analyses were done using the equation $\sum_{\mathrm{j}} \mathrm{s}_{\mathrm{ij}}=\sum_{\mathrm{j}} \mathrm{e}^{\left(\frac{\mathrm{R}_{\mathrm{ij}}^{0}-\mathrm{R}_{\mathrm{ij}}}{\mathrm{B}}\right)}$, with parameters $R_{(\mathrm{V}-\mathrm{N})}=1.86 \AA, R_{(\mathrm{V}-\mathrm{O})}=1.803 \AA, R_{(\mathrm{V}-\mathrm{F})}=1.71 \AA$, $B=0.37 \AA$. $^{1,2}$ 
Table S4. Comparison some of selected structural features in anions $\left[\mathrm{VOF}_{4}\right]^{-}, 3\left[\mathrm{VOF}_{4}(\mathrm{THF})\right]^{-}$and $\left[\mathrm{VOF}_{4}(\mathrm{Py})\right]^{-}$.

\begin{tabular}{|c|c|c|c|c|}
\hline & $\begin{array}{l}{\left[\mathrm{VOF}_{4}\right]^{-}} \\
\text {domain A }\end{array}$ & {$\left[\mathrm{VOF}_{4}(\mathrm{THF})\right]^{-}$} & $\begin{array}{l}{\left[\mathrm{VOF}_{4}(\mathrm{Py})\right]^{-}} \\
\text {subunit } 1\end{array}$ & $\begin{array}{l}{\left[\mathrm{VOF}_{4}(\mathrm{Py})\right]^{-}} \\
\text {subunit } 2\end{array}$ \\
\hline \multicolumn{5}{|c|}{ Bond length $[\AA]$} \\
\hline $\mathrm{V}=\mathrm{O}$ & $1.560(2)$ & $1.561(2)$ & $1.570(2)$ & $1.574(2)$ \\
\hline $\mathrm{V}-\mathrm{F} 1$ & $1.776(2)$ & $1.819(1)$ & $1.840(1)$ & $1.832(1)(\mathrm{F} 5)$ \\
\hline $\mathrm{V}-\mathrm{F} 2$ & $1.815(2)$ & $1.805(1)$ & $1.804(1)$ & $1.805(1)(\mathrm{F} 6)$ \\
\hline $\mathrm{V}-\mathrm{F} 3$ & $1.807(2)$ & $1.806(1)$ & $1.831(1)$ & $1.821(1)(\mathrm{F} 7)$ \\
\hline $\mathrm{V}-\mathrm{F} 4$ & $1.794(2)$ & $1.821(1)$ & $1.811(1)$ & $1.815(1)(\mathrm{F} 8)$ \\
\hline $\mathrm{V}-\mathrm{X}_{\text {ligand }}[\mathrm{c}]$ & $\mathrm{NA}^{[\mathrm{b}]}$ & $\begin{array}{l}2.403(7)(\mathrm{O} 30 \mathrm{~A}) \\
2.464(6)(\mathrm{O} 30 \mathrm{~B})\end{array}$ & $2.399(2)$ & $2.406(2)$ \\
\hline \multicolumn{5}{|c|}{ Cation displacement $[\AA]$} \\
\hline $\mathrm{V}-$ Plane $_{\mathrm{F}}[\mathrm{c}]$ & 0.446 & 0.309 & 0.291 & 0.288 \\
\hline \multicolumn{5}{|l|}{ Angle $\left[{ }^{\circ}\right]$} \\
\hline $\mathrm{O}=\mathrm{V}-\mathrm{F} 1$ & $107.0(1)$ & $99.64(7)$ & $99.45(9)$ & $98.62(9)(\mathrm{F} 5)$ \\
\hline $\mathrm{O}=\mathrm{V}-\mathrm{F} 2$ & $104.5(1)$ & $100.21(9)$ & $99.49(8)$ & 98.07(8) (F6) \\
\hline $\mathrm{O}=\mathrm{V}-\mathrm{F} 3$ & $103.1(1)$ & $100.53(7)$ & $99.13(9)$ & $99.34(9)(\mathrm{F} 7)$ \\
\hline $\mathrm{O}=\mathrm{V}-\mathrm{F} 4$ & $103.4(1)$ & $98.69(8)$ & $98.61(9)$ & $100.40(7)(\mathrm{F} 8)$ \\
\hline $\mathrm{F}-\mathrm{V}-\mathrm{F}_{\text {trans }}$ & $\begin{array}{l}149.8(1)(\mathrm{F} 1 \mathrm{~A}, \mathrm{~F} 3 \mathrm{~A}) \\
152.08(9)(\mathrm{F} 2 \mathrm{~A}, \mathrm{~F} 4 \mathrm{~A})\end{array}$ & $\begin{array}{l}159.78(6)(\mathrm{F} 1, \mathrm{~F} 3) \\
161.05(7)(\mathrm{F} 2, \mathrm{~F} 4)\end{array}$ & $\begin{array}{l}161.37(6)(\mathrm{F} 1, \mathrm{~F} 3) \\
161.87(7)(\mathrm{F} 2, \mathrm{~F} 4)\end{array}$ & $\begin{array}{l}161.85(7)(\mathrm{F} 5, \mathrm{~F} 7) \\
161.53(6)(\mathrm{F} 6, \mathrm{~F} 8)\end{array}$ \\
\hline $\mathrm{O}=\mathrm{V}-\mathrm{X}_{\text {ligand }}$ & $\mathrm{NA}^{[\mathrm{b}]}$ & $\begin{array}{l}171.3(2)(\mathrm{O} 30 \mathrm{~A}) \\
175.1(2)(\mathrm{O} 30 \mathrm{~B})\end{array}$ & 179.61(9) & $176.78(8)$ \\
\hline
\end{tabular}

[a] $\mathrm{X}$ represents electron donating moiety of the ligand ( $\mathrm{O}$ for THF, $\mathrm{N}$ for Py).

${ }^{[b]}$ Not applicable, as the sixth coordination site of the anion is empty.

${ }^{[c]}$ Distance of $\mathrm{V}$ from the plane of $\mathrm{F}$ atoms. 
Table S5. Selected examples of structurally characterized compounds with vanadium oxofluorido anions.

\begin{tabular}{|c|c|c|c|c|c|c|c|c|c|c|}
\hline \multirow{2}{*}{ Compound } & \multirow[t]{2}{*}{$\mathrm{V}-\mathrm{O}$} & \multirow{2}{*}{$\begin{array}{l}\text { Axial } \\
\text { ligand }\end{array}$} & \multirow{2}{*}{$\begin{array}{l}\text { Bond length of axial } \\
\text { ligand }[\AA]\end{array}$} & \multirow{2}{*}{$\begin{array}{l}\mathrm{O}-\mathrm{V}-\mathrm{O} / \mathrm{F}_{\text {trans }} \\
{\left[^{\circ}\right]}\end{array}$} & \multirow{2}{*}{$\begin{array}{l}\text { Average bond length of } \\
\text { equatorial ligands }[\AA]\end{array}$} & \multirow{2}{*}{$\begin{array}{ll}\text { Average (range } & \text { of } \\
\left.\mathrm{O}-\mathrm{V}-\mathrm{F} \text { angles }\left[{ }^{\circ}\right]\right)\end{array}$} & \multirow{2}{*}{ Structural unit } & \multicolumn{2}{|c|}{ Displacement $[\AA]$} & \multirow{2}{*}{ Reference } \\
\hline & & & & & & & & Reported & Calculated & \\
\hline$\left[\mathrm{enH}_{2}\right]\left[\mathrm{VOF}_{5}\right]^{[a]}$ & $1.54(1)$ & $\mathrm{F}$ & $2.10(1)$ & $177.6(5)$ & $\begin{array}{l}1.80 \\
(1.75(1)-1.85(1))\end{array}$ & $\begin{array}{l}98.2 \\
(96.1(5)-101.0(6))\end{array}$ & Monomer & 0.27 & 0.258 & [4] \\
\hline $\mathrm{Na}_{2}\left[\mathrm{VOF}_{5}\right]$ & $1.607(6)$ & $\mathrm{F}$ & $2.140(6)$ & $180^{\text {calc }}$ & $\begin{array}{l}1.892 \\
(1.892(3))\end{array}$ & $\begin{array}{l}98.1 \\
(98.1(2))\end{array}$ & Monomer & 0.27 & 0.266 & [5] \\
\hline$[\mathrm{PyH}]_{2}\left[\mathrm{Cu}(\mathrm{Py})_{4}\left(\mathrm{VOF}_{5}\right)_{2}\right]^{[\mathrm{b}]}$ & $1.596(2)$ & $\mathrm{F}$ & $2.084(2)$ & $178.27(8)^{\text {calc }}$ & $\begin{array}{l}1.837 \\
(1.811(2)-1.883(2))\end{array}$ & $\begin{array}{l}96.57 \\
(95.71(8)-97.20(9))\end{array}$ & Monomer & & 0.211 & [6] \\
\hline$\left[4,4^{\prime}-\mathrm{bpyH}_{2}\right]\left[\mathrm{VOF}_{5}\right]^{[\mathrm{c}]}$ & $1.587(3)$ & $\mathrm{F}$ & $2.193(2)$ & $179.6(1)$ & $\begin{array}{l}1.819 \\
(1.802(2)-1.826(2))\end{array}$ & $\begin{array}{l}97.9 \\
(96.7(1)-98.9(1))\end{array}$ & Monomer & & 0.250 & [7] \\
\hline$\left[\mathrm{enH}_{2}\right]\left[\mathrm{VOF}_{4}\left(\mathrm{H}_{2} \mathrm{O}\right)\right]^{[\mathrm{a}]}$ & $1.577(3)$ & $\mathrm{H}_{2} \mathrm{O}$ & $2.333(2)$ & $177.3(1)$ & $\begin{array}{l}1.808 \\
(1.769(2)-1.858(2))\end{array}$ & $\begin{array}{l}99.1 \\
(98.0(1)-99.7(1)\end{array}$ & Monomer & 0.33 & 0.286 & [9] \\
\hline$\left[\mathrm{pipH}_{2}\right]_{3}\left[\mathrm{~V}_{2} \mathrm{O}_{2} \mathrm{~F}_{8}\right]\left[\mathrm{VOF}_{4}\left(\mathrm{H}_{2} \mathrm{O}\right)\right]_{2}^{[\mathrm{d}]}$ & $1.578(3)$ & $\mathrm{H}_{2} \mathrm{O}$ & $2.274(3)$ & $178.0(1)$ & $\begin{array}{l}1.828 \\
(1.795(2)-1.843(2))\end{array}$ & $\begin{array}{l}99.0 \\
(98.1(1)-100.7(1))\end{array}$ & Monomer & & 0.286 & [10] \\
\hline \multirow{3}{*}{$\mathrm{Cs}\left[\mathrm{NMe}_{4}\right]\left[\mathrm{V}_{2} \mathrm{O}_{2} \mathrm{~F}_{8}\left(\mathrm{H}_{2} \mathrm{O}\right)\right]^{[\mathrm{e}]}$} & $1.581(4)$ & $\mathrm{F}$ & $2.322(3)$ & $179.4(2)$ & 1.815 & 99.9 & \multirow{3}{*}{$\begin{array}{l}\text { Corner-shared } \\
\text { dimer }\end{array}$} & & 0.314 & \multirow{3}{*}{ [11] } \\
\hline & & & & & $\begin{array}{l}(1.813(3)-1.816(3)) \\
1.822\end{array}$ & $\begin{array}{l}(99.8(1)-100.0(1)) \\
98.5\end{array}$ & & & & \\
\hline & $1.575(4)$ & $\mathrm{H}_{2} \mathrm{O}$ & $2.294(4)$ & $179.0(2)$ & $(1.804(2)-1.864(3))$ & $(96.5(2)-100.4(1))$ & & & 0.321 & \\
\hline $\mathrm{Cs}\left[\mathrm{VOF}_{4}\right]$ & $1.53(1)$ & $\mathrm{F}$ & $2.31(1)$ & $178.8(7)$ & $\begin{array}{l}1.787 \\
(1.78(1)-1.79(1))\end{array}$ & $\begin{array}{l}100.4 \\
(98.1(7)-102.0(7))\end{array}$ & 1D chain & 0.326 & 0.325 & [12] \\
\hline $\mathrm{K}\left[\mathrm{VOF}_{4}\right]$ & $1.572(7)$ & $\mathrm{F}$ & $2.333(6)$ & $178.4(5)^{\circ}$ & $\begin{array}{l}1.814 \\
(1.786(7)-1.875(6))\end{array}$ & $\begin{array}{l}100.0 \\
(98.4(5)-100.9(5))\end{array}$ & 1D chain & 0.31 & 0.315 & \\
\hline$[\mathrm{EMIm}]\left[\mathrm{VOF}_{4}\right]^{[\mathrm{ff}]}$ & $1.575(1)$ & $\mathrm{F}$ & $2.3786(7)$ & $173.15(5)$ & $\begin{array}{l}1.8203 \\
(1.7883(8)-1.8633(7)) \\
\end{array}$ & $\begin{array}{l}100.74 \\
(98.52(4)-103.64(5)) \\
\end{array}$ & $\begin{array}{l}\text { Edge-shared } \\
\text { dimer }\end{array}$ & & 0.333 & [13] \\
\hline $\mathrm{Pb}\left[\mathrm{V}_{2} \mathrm{O}_{2} \mathrm{~F}_{8}\right]$ & $1.564(5)$ & $\mathrm{F}$ & $2.401(4)$ & $175.4(2)$ & $\begin{array}{l}1.830 \\
(1.804(4)-1.871(4)) \\
\end{array}$ & $\begin{array}{l}102.2 \\
(100.3(2)-104.4(2)) \\
\end{array}$ & $\begin{array}{l}\text { Edge-shared } \\
\text { dimer }\end{array}$ & & 0.386 & [8] \\
\hline $\mathrm{Pb}_{3} \mathrm{~F}\left[\mathrm{~V}_{4} \mathrm{O}_{3} \mathrm{~F}_{18}\right]$ & $1.55(2)$ & $\mathrm{F}$ & $2.38(2)$ & $174(1)$ & $\begin{array}{l}1.84 \\
(1.77(2)-1.88(2))\end{array}$ & $\begin{array}{l}100.4^{\text {calculated }} \\
(97.4-103.0)^{\text {calculated }}\end{array}$ & $\begin{array}{l}\text { Corner-shared } \\
\text { tetramer }\end{array}$ & & 0.330 & [8] \\
\hline $\mathrm{Ag}[\mathrm{VOF} 4]$ & $1.571(3)$ & $\mathrm{F}$ & $2.287(2)$ & 179.2(1) & $\begin{array}{l}1.824 \\
(1.798(2)-1.857(2))\end{array}$ & $\begin{array}{l}100.1 \\
(99.3(1)-101.4(1))\end{array}$ & 1D chain & & 0.320 & [14] \\
\hline$\left[\mathrm{NH}_{3} \mathrm{OH}\right]\left[\mathrm{VOF}_{4}\right]$ & $1.566(1)$ & $\mathrm{F}$ & $2.3306(7)$ & 178.84(4) & $\begin{array}{l}1.8262 \\
(1.8094(7)-1.8540(8))\end{array}$ & $\begin{array}{l}99.96 \\
(99.20(4)-101.07(4))\end{array}$ & 1D chain & & 0.316 & {$[15]$} \\
\hline
\end{tabular}

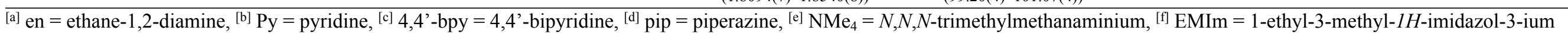




\section{S2. Comparison of experimental and DFT calculation data}

Table S6. Comparison between the PBE-D3/plane-wave calculated and the experimentally determined lattice parameters and unit-cell volumes of $\left[\left(\mathrm{L}^{\text {Dipp }}\right) \mathrm{H}\right]\left[\mathrm{VOF}_{4}(\mathrm{THF})\right] \quad(\mathbf{1}), \quad\left[\left(\mathrm{L}^{\mathrm{Dipp}}\right) \mathrm{H}\right]\left[\mathrm{VOF}_{4}(\mathrm{Py})\right] \quad$ (2), and $\left[\mathrm{L}^{\mathrm{PPh} 3 \mathrm{Me}}\right]\left[\mathrm{NbOCl}_{4}\left(\mathrm{CH}_{3} \mathrm{CN}\right)\right]$ crystal structures. The calculated parameters for the hypothetical $\left[\left(\mathrm{L}^{\mathrm{Dipp}}\right) \mathrm{H}\right]\left[\mathrm{VOF}_{4}\left(\mathrm{H}_{2} \mathrm{O}\right)\right]$ and hypothetical $\left[\mathrm{L}^{\mathrm{PPh} 3 \mathrm{Me}}\right]\left[\mathrm{VOCl}_{4}\left(\mathrm{CH}_{3} \mathrm{CN}\right)\right]$ crystal structures are also reported, which were obtained by replacing $\mathrm{Nb}$ with $\mathrm{V}$ in $\left[\mathrm{L}^{\mathrm{PPh} 3 \mathrm{Me}}\right]\left[\mathrm{NbOCl}_{4}\left(\mathrm{CH}_{3} \mathrm{CN}\right)\right]$ and by replacing THF with $\mathrm{H}_{2} \mathrm{O}$ in $\left[\left(\mathrm{L}^{\mathrm{Dipp}}\right) \mathrm{H}\right]\left[\mathrm{VOF}_{4}(\mathrm{THF})\right](\mathbf{1})$ and reoptimizing the crystal structures with the variable-cell relaxations.

\begin{tabular}{|c|c|c|c|c|c|c|c|c|c|c|}
\hline & \multicolumn{2}{|c|}{$\begin{array}{c}{\left[\left(\mathrm{L}^{\mathrm{Dipp}}\right) \mathrm{H}\right]} \\
{\left[\mathrm{VOF}_{4}(\mathrm{THF})\right](\mathbf{1})}\end{array}$} & \multicolumn{2}{|c|}{$\begin{array}{c}{\left[\left(\mathrm{L}^{\text {Dipp }}\right) \mathrm{H}\right]} \\
{\left[\mathrm{VOF}_{4}(\mathrm{Py})\right](2)}\end{array}$} & \multicolumn{2}{|c|}{$\begin{array}{c}{\left[\left(\mathrm{L}^{\text {Dipp }}\right) \mathrm{H}\right]} \\
{\left[\mathrm{VOF}_{4}\left(\mathrm{H}_{2} \mathrm{O}\right)\right]}\end{array}$} & \multicolumn{2}{|c|}{$\begin{array}{c}{\left[\mathrm{L}^{\mathrm{PPh} 3 \mathrm{Me}}\right]} \\
{\left[\mathrm{VOCl}_{4}\left(\mathrm{CH}_{3} \mathrm{CN}\right)\right]}\end{array}$} & \multicolumn{2}{|c|}{$\begin{array}{c}{\left[\mathrm{L}^{\mathrm{PPh} 3 \mathrm{Me}}\right]} \\
{\left[\mathrm{NbOCl}_{4}\left(\mathrm{CH}_{3} \mathrm{CN}\right)\right]}\end{array}$} \\
\hline & PBE-D3 & Expt. ${ }^{[a]}$ & PBE-D3 & Expt. & PBE-D3 & Expt. & PBE-D3 & Expt. & PBE-D3 & $\operatorname{Expt}^{[\mathrm{cc}]}$ \\
\hline$a[\AA]]$ & 12.63 & 12.66 & 12.52 & 12.57 & 12.61 & 1 & 11.40 & 1 & 11.55 & 11.74 \\
\hline$b[\AA]$ & 12.66 & 12.78 & 12.65 & 12.72 & 12.73 & I & 11.13 & / & 11.24 & 11.30 \\
\hline$c[\AA]$ & 19.56 & 19.61 & 20.57 & 20.56 & 19.33 & I & 9.38 & I & 9.42 & 9.47 \\
\hline$\alpha\left[^{\circ}\right]$ & 90.0 & 90.0 & 88.7 & 88.9 & 90.0 & I & 97.8 & / & 97.8 & 97.7 \\
\hline$\beta\left[^{\circ}\right]$ & 91.9 & 93.4 & 80.8 & 81.2 & 90.0 & I & 94.0 & I & 93.7 & 93.6 \\
\hline$\gamma\left[^{\circ}\right]$ & 90.0 & 90.0 & 87.1 & 87.1 & 90.0 & I & 79.0 & l & 78.6 & 78.6 \\
\hline$V\left[\AA^{3}\right]$ & 3127 & 3165 & 3210 & 3244 & 3103 & 1 & 1157 & I & 1187 & 1219 \\
\hline
\end{tabular}

[a] Disordered.

[b] No experimental data available.

[c] Reference ${ }^{16}$. 

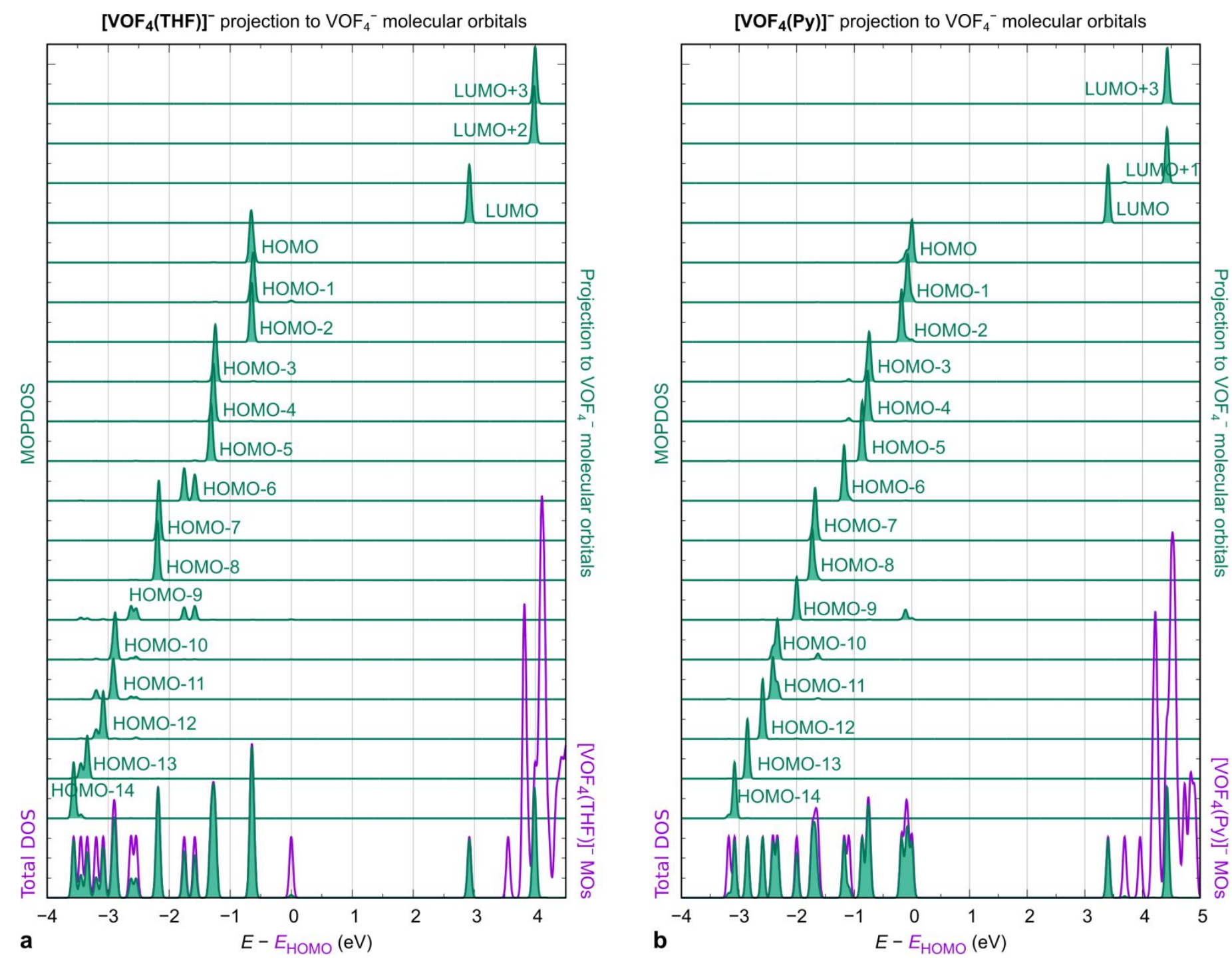

Figure S1: Density of states (DOS) analyses of the standalone (a) $\left[\mathrm{VOF}_{4}(\mathrm{THF})\right]^{-}$and (b) $\left[\mathrm{VOF}_{4}(\mathrm{Py})\right]^{-}$complexes. The purple curves at the bottom of the plots represent the total DOS, whereas the superposed green curves are the DOS projections to the $\mathrm{VOF}_{4}^{-}$fragment. Above these curves the DOS is projected to individual molecular orbitals of the $\mathrm{VOF}_{4}^{-}$anion. The labels HOMO- $n$ and LUMO $+m$ stand for the $n$th orbital below HOMO and $m$ th orbital above LUMO, respectively. 
$\mathrm{VOF}_{4}^{-}$

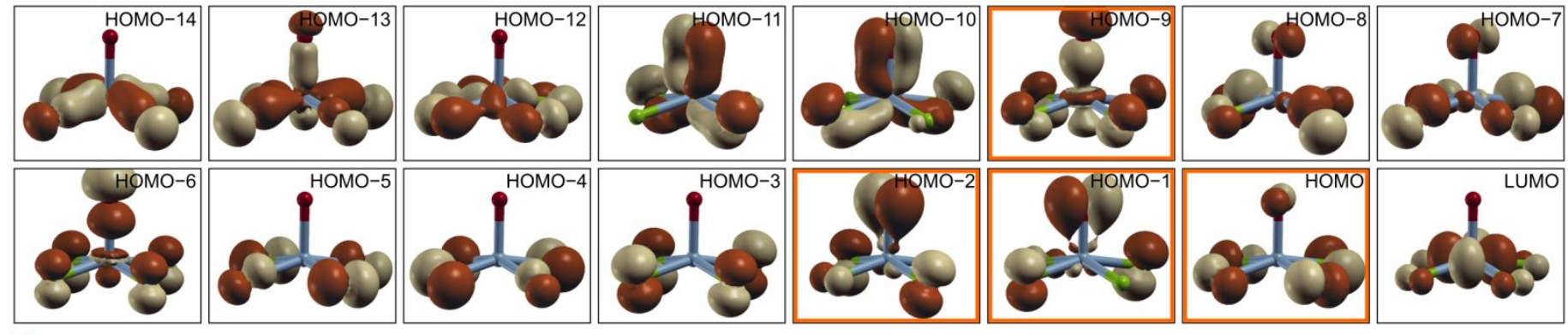

Py
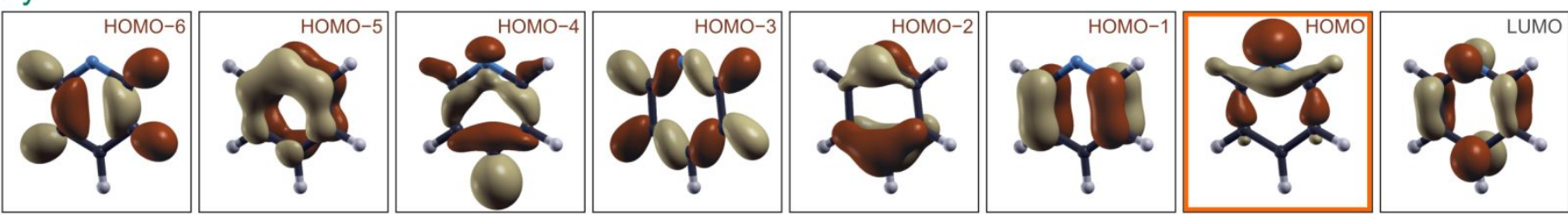

\section{$\left[\mathrm{VOF}_{4}(\mathrm{Py})\right]^{-}$}

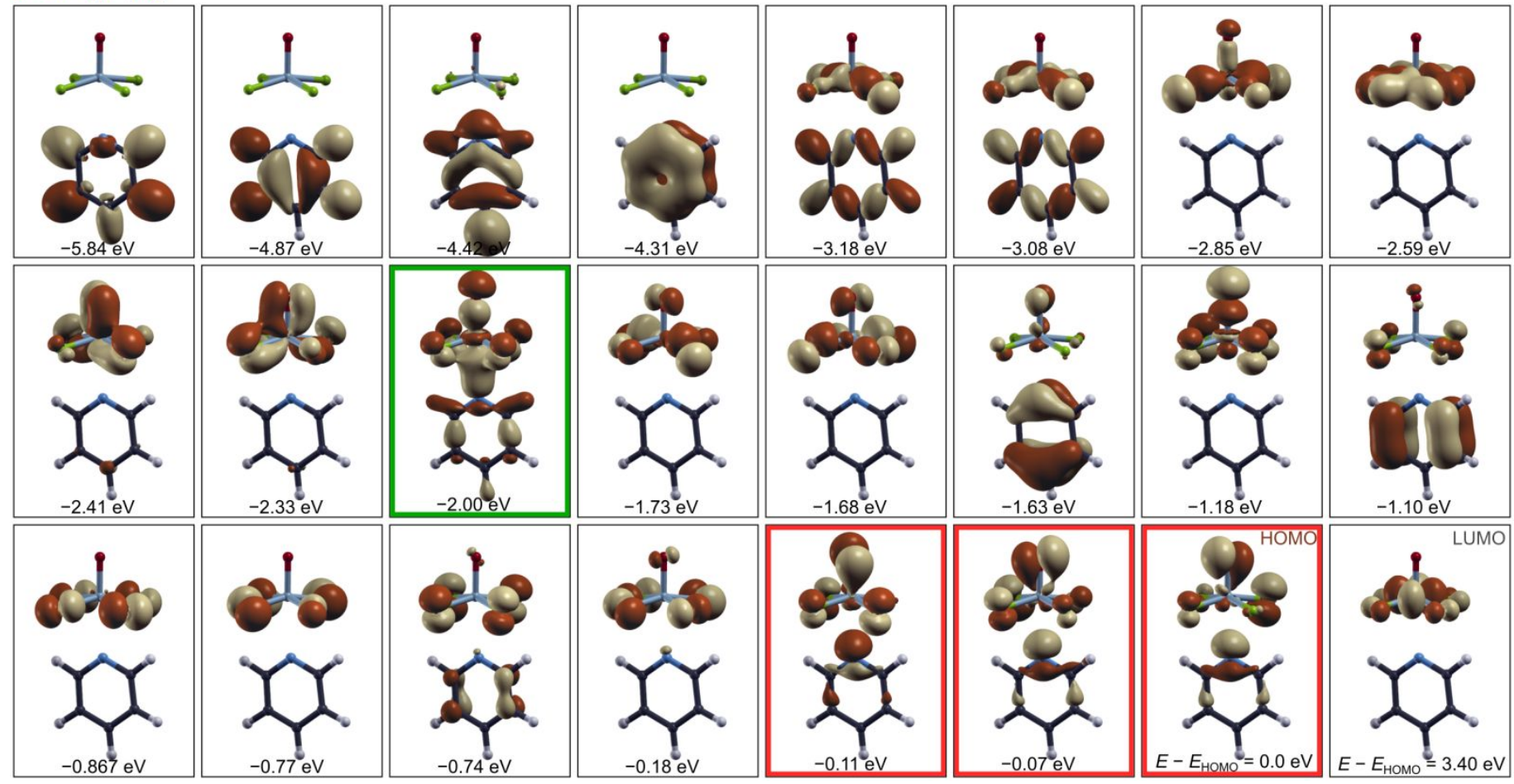

Figure S2: A subset of PBE-D3 calculated signed molecular orbital densities, $\operatorname{sgn}\left(\psi_{\mathrm{i}}(\mathbf{r})\right)\left|\psi_{\mathrm{i}}(\mathbf{r})\right|^{2}$, of the $\mathrm{VOF}_{4}^{-}$anion, the Py ligand, and the standalone $\left[\mathrm{VOF}_{4}(\mathrm{Py})\right]^{-}$complex. The label HOMO- $n$ stands for the $n$th orbital below HOMO. There is only one bonding molecular orbital between $\mathrm{VOF}_{4}$ and $\mathrm{Py}$, which is highlighted with green rectangle, whereas molecular orbitals between the two fragments that display more anti-bonding character are highlighted by red rectangles. Molecular orbitals of the individual $\mathrm{VOF}_{4}^{-}$and Py fragments that are predominantly involved in the highlighted states are marked with orange rectangles. 


\section{S5. Some electronic parameters of ligands and metal centers}

Vertical ionization potentials $(I)$ and vertical electron affinities $(A)$ were calculated as:

$$
I=\left[E_{X^{+}}-E_{X}\right]_{v} \text { and } A=\left[E_{X}-E_{X^{-}}\right]_{v^{\prime}}
$$

where $X$ stands for the neutral molecule and $X^{+}$and $X^{-}$are the corresponding cation and anion, respectively, that correspond to the $X \rightarrow X^{+}+\mathrm{e}^{-}$and $X+\mathrm{e}^{-} \rightarrow X^{-}$reactions. To ensure constant external potential $v$, the molecular geometries of $X^{+}$and $X^{-}$were kept fixed to that of $\mathrm{X}$. The Mulliken electronegativities $(\chi)$ and chemical hardnesses $(\eta)$ were calculated from $I$ and $A$ as:

$$
\chi=\frac{I+A}{2} \text { and } \eta=\frac{I-A}{2} .
$$

The PBE-D3/def2TZVP calculated values of $I, A, \chi$, and $\eta$ of the considered ligands are given in Table S7.

Table S7. PBE-D3/def2TZVP calculated vertical ionization potential ( $I)$, vertical electron affinity $(A)$, Mulliken electronegativity $(\chi)$, and chemical hardness $(\eta)$ of the considered ligands.

\begin{tabular}{ccccc}
\hline & $\begin{array}{c}I \\
(\mathrm{eV})\end{array}$ & $\begin{array}{c}A \\
(\mathrm{eV})\end{array}$ & $\begin{array}{c}\chi \\
(\mathrm{eV})\end{array}$ & $\begin{array}{c}\eta \\
(\mathrm{eV})\end{array}$ \\
\hline N-ligands & & & & \\
$\mathrm{CH}_{3} \mathrm{CN}$ & 12.05 & -2.12 & 4.97 & 7.09 \\
$\mathrm{NH}_{3}$ & 10.89 & -2.51 & 4.19 & 6.70 \\
$\mathrm{Py}$ & 9.31 & -1.03 & 4.14 & 5.17 \\
$\mathrm{ABCO}$ & 7.64 & -1.84 & 2.90 & 4.74 \\
O-ligands & & & & \\
$\mathrm{H} \mathrm{H}_{2} \mathrm{O}$ & 12.65 & -2.52 & 5.06 & 7.58 \\
$\mathrm{OCCH}$ & 9.72 & -1.39 & 4.16 & 5.56 \\
$\mathrm{THF}$ & 9.38 & -2.31 & 3.54 & 5.84 \\
\hline
\end{tabular}

The ionization potential and electron affinity and, in turn, electronegativity and chemical hardness of the $\mathrm{V}(+5)$ and $\mathrm{Nb}(+5)$ metal centers were roughly estimated by two different approaches - note that here trends and not specific values are of interest. In the first approach, the formal oxidation state of +5 is equated with the actual charge of 5+ (this approach is named as " $\mathrm{M}(+5)=\mathrm{M}^{5+}$ approximation"). In this case, the ionization potential of $\mathrm{V}^{5+}$ is given by the sixth ionization potential of $\mathrm{V}$ that corresponds to the $\mathrm{V}^{5+} \rightarrow \mathrm{V}^{6+}+\mathrm{e}^{-}$reaction, whereas the electron affinity of $\mathrm{V}^{5+}$ corresponds to $\mathrm{V}^{4+} \rightarrow \mathrm{V}^{5+}+\mathrm{e}^{-}$and is therefore given by the fifth ionization potential of $\mathrm{V}$. In the second approach, the calculated Bader charge of $\mathrm{V}(5+)$ center of $\mathrm{VOF}_{4}^{-}$is utilized, which equals 2.3. This charge best corresponds to $\mathrm{V}^{2+}$ ion, hence the corresponding $I$ and $A$ values are approximated by the third and second ionization potential of $\mathrm{V}$, respectively (this approach is named as "M(+5) $=\mathrm{M}^{2+}$ approximation"). The case of $\mathrm{Nb}(5+)$ is treated analogously. The experimental values for the first six ionization potentials of $\mathrm{V}$ and $\mathrm{Nb}$ are tabulated in Table $\mathrm{S} 8$, whereas the $I, A, \chi$, and $\eta$ values of $\mathrm{V}(+5)$ 
and $\mathrm{Nb}(+5)$, deduced from the two approximations, are given in Table S9. It can be seen that both approaches univocally show that $\mathrm{V}(+5)$ is more electronegative and chemically harder than $\mathrm{Nb}(5+)$ thus suggesting that $\mathrm{V}(+5)$ is a harder Lewis acid than $\mathrm{Nb}(+5)$.

Table S8. Experimentally determined first six ionization potentials of V and Nb, Data taken from ref. ${ }^{17}$

\begin{tabular}{lcccccc}
\hline & \multicolumn{6}{c}{ Ionization potentials $(\mathrm{eV})$} \\
& 1st & 2nd & 3rd & 4th & 5th & 6th \\
\hline $\mathrm{V}$ & 6.7 & 14.7 & 29.3 & 46.7 & 65.3 & 128.1 \\
$\mathrm{Nb}$ & 6.8 & 14.3 & 25.0 & 38.3 & 50.5 & 102.1 \\
\hline
\end{tabular}

Table S9. Approximate vertical ionization potential $(I)$, vertical electron affinity $(A)$, Mulliken electronegativity $(\chi)$, and chemical hardness $(\eta)$ of $\mathrm{V}(+5)$ and $\mathrm{Nb}(+5)$ metal centers estimated with aid of the " $\mathrm{V}(+5)=\mathrm{V}^{5+}$ " and " $\mathrm{V}(+5)=\mathrm{V}^{2+}$ " approximations and the experimental values for the ionization potentials of $\mathrm{V}$ and $\mathrm{Nb}$, taken from Table $\mathrm{S} 8$.

\begin{tabular}{|c|c|c|c|c|}
\hline & $\begin{array}{c}I \\
(\mathrm{eV})\end{array}$ & $\begin{array}{c}A \\
(\mathrm{eV})\end{array}$ & $\begin{array}{c}\chi \\
(e V)\end{array}$ & $\begin{array}{c}\eta \\
(\mathrm{eV})\end{array}$ \\
\hline \multicolumn{5}{|c|}{$\mathrm{V}(+5)=\mathrm{V}^{5+}$ approximation } \\
\hline & $I=I^{6 \text { th }}$ & $A=I^{5 \text { th }}$ & & \\
\hline $\mathrm{V}^{5+}$ & 128.1 & 65.3 & 96.7 & 31.4 \\
\hline $\mathrm{Nb}^{5+}$ & 102.1 & 50.5 & 76.3 & 25.8 \\
\hline \multicolumn{5}{|c|}{$\mathrm{V}(+5)=\mathrm{V}^{2+}$ approximation } \\
\hline & $I=I^{3 \mathrm{rd}}$ & $A=I^{2 \text { nd }}$ & & \\
\hline $\mathrm{V}^{2+}$ & 29.3 & 14.7 & 22.0 & 7.3 \\
\hline $\mathrm{Nb}^{2+}$ & 25.0 & 14.3 & 19.7 & 5.4 \\
\hline
\end{tabular}


S6. Estimation of the $\mathrm{X} \cdots \mathrm{H}-\mathrm{C}$ bond strengths
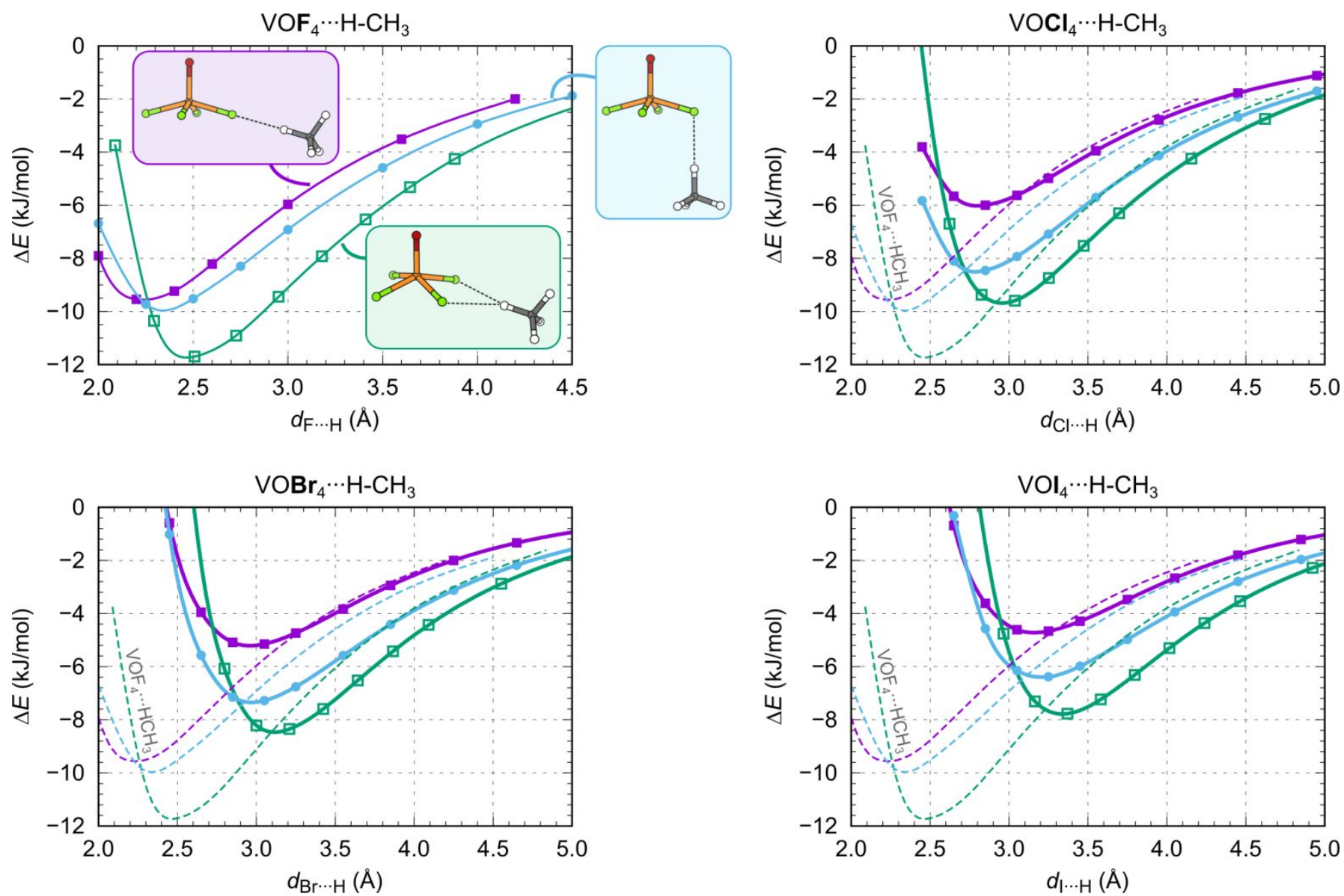

Figure S3. PBE-D3/def2TZVP calculated interaction energies between methane $\left(\mathrm{CH}_{4}\right)$ and $\left[\mathrm{VOX}_{4}\right]^{-}$anions $(\mathrm{X}=\mathrm{F}, \mathrm{Cl}$, $\mathrm{Br}$, and I) in various geometries as a function of the $\mathrm{F} \cdots \mathrm{H}$ distance. These calculations were used to estimate the strength of the linear and bifurcated $\mathrm{X} \cdots \mathrm{H}-\mathrm{C}$ bonds. 


\section{S7. References}

${ }^{1}$ Brown I. D.; Altermatt D.; Bond-Valence Parameters Obtained from a Systematic Analysis of the Inorganic Crystal Structure Database, Acta Crystallogr. Sect. B 1985, 41(4), 244-247.

${ }^{2}$ Brese N. E.; O’Keeffe M.; Bond-Valence Parameters for Solids, Acta Crystallogr. Sect. B, 1991, 47(2), pp. $192-197$.

${ }^{3}$ Zupanek Ž.; Tramšek M.; Kokalj A.; Tavčar G.; Reactivity of $\mathrm{VOF}_{3}$ with N-Heterocyclic Carbene and Imidazolium Fluoride: Analysis of Ligand- $\mathrm{VOF}_{3}$ Bonding with Evidence of a Minute $\pi$ Back-Donation of Fluoride. Inorg. Chem. 2018, 57 (21), 1386613879.

${ }^{4}$ Rieskamp H.; Mattes R.; Fluorreiche Oxofluorovanadate(V): Die Kristallstruktur von [enH $\left.\mathrm{e}_{2}\right]\left[\mathrm{VOF}_{5}\right]$, Zeitschrift für Naturforsch. $B, 1976,31$ (11), 1453-1455.

5 Stomberg R.; Crystal Structure of Sodium Pentafluorooxovanadate(V), $\mathrm{Na}_{2}\left[\mathrm{VF}_{5} \mathrm{O}\right]$, and Potassium catena-mu-Fluorodifluorodioxovanadate $(\mathrm{V}), \mathrm{K}_{2 \mathrm{n}}\left[\left(\mathrm{VF}_{3} \mathrm{O}_{2}\right)_{\mathrm{n}}\right]$, Two Decomposition Products in the System $\mathrm{V}_{2} \mathrm{O}_{5} /(\mathrm{MF}, \mathrm{HF}) / \mathrm{H}_{2} \mathrm{O}_{2} / \mathrm{H}_{2} \mathrm{O}$, Acta Chem . Scand., 1986, 40A, 325-330.

${ }^{6}$ Welk M. E.; Norquist A. J.; Stern C. L.; Poeppelmeier K. R.; The Structure-Directing Properties of [VOF 5$]^{2-}$, Inorg. $_{\text {Chem. }}$ 2000, 39 (18), 3946-3947.

${ }^{7}$ Gautier R.; Chang K. B.; Poeppelmeier K. R.; On the Origin of the Differences in Structure Directing Properties of Polar Metal Oxyfluoride $\left[\mathrm{MO}_{\mathrm{x}} \mathrm{F}_{6-\mathrm{x}}\right]^{2-}(\mathrm{x}=1,2)$ Building Units, Inorg. Chem., 2015, 54, (4), 1712-1719.

8 Lozinšek M.; Goreshnik E.; Žemva B.; Lead Fluoridooxidovanadates(V), $\left.\mathrm{Pb}\left(\mathrm{V}_{2} \mathrm{O}_{2} \mathrm{~F}_{8}\right), \mathrm{Pb}_{(\mathrm{VOF}}\right)$, and Mixed Valent Fluoridooxidovanadate( $\mathrm{IV}, \mathrm{V}), \mathrm{Pb}_{3} \mathrm{~F}\left(\mathrm{~V}_{4} \mathrm{O}_{3} \mathrm{~F}_{18}\right)$, Zeitschrift für Anorg. und Allg. Chemie, 2012, 638 (12-13), 2123-2128.

${ }^{9}$ Rieskamp H.; Mattes R.; Oxotetrafluoro-vanadate(V): Die Kristallstruktur von $\left[\mathrm{enH}_{2}\right]\left[\mathrm{VOF}_{4}\left(\mathrm{H}_{2} \mathrm{O}\right)\right]_{2}$, Zeitschrift für Naturforsch. $B$ 1976, 31(5), 541-543.

10 Aldous D. W.; Stephens N. F.; Lightfoot P.; The role of temperature in the solvothermal synthesis of hybrid vanadium oxyfluorides, Dalt. Trans. 2007 (37), 4207-4213.

11 Hilbers M.; Leimkühler M.; Mattes R.; Zwei-und dreikernige Fluorooxoanionen des Vanadins. Kristallstrukturen von $\mathrm{Cs}\left(\mathrm{NMe}_{4}\right)\left[\mathrm{V}_{2} \mathrm{O}_{2} \mathrm{~F}_{8}\left(\mathrm{H}_{2} \mathrm{O}\right)\right]$ und $(\mathrm{Na}, \mathrm{K})\left(\mathrm{NMe}_{4}\right)_{2}\left[\mathrm{~V}_{3} \mathrm{O}_{3} \mathrm{~F}_{12}\right]$, Zeitschrift für Naturforsch. B 1989, 44 (4), $383-388$.

12 Bushnell G. W.; Moss K. C.; The Crystal Structure of Caesium Oxotetrafluorovanadate(V), Can. J. Chem. 1972, 50 (22), 3700 3705 .

${ }^{13}$ Kanatani T.; Matsumoto K.; Hagiwara R.; Syntheses and Physicochemical Properties of Low-Melting Salts Based on VOF ${ }_{4}^{-}$and $\mathrm{MoOF}_{5}^{-}$, and the Molecular Geometries of the Dimeric $\left(\mathrm{VOF}_{4}^{-}\right)_{2}$ and $\mathrm{Mo}_{2} \mathrm{O}_{4} \mathrm{~F}_{6}{ }^{2-}$ Anions, Eur. J. Inorg. Chem., 2010, 2010 (7), 10491055 .

${ }^{14}$ Lozinšek M.; Goreshnik E.; Žemva B.; Silver(I) Tetrafluoridooxidovanadate(V) - Ag[VOF 4 , Acta Chim. Slov. 2014, 61 (3), 542547.

${ }^{15}$ Lozinšek M.; Hydroxylammonium Tetrafluoridooxidovanadate(V) - $\left(\mathrm{NH}_{3} \mathrm{OH}\right)\left[\mathrm{VOF}_{4}\right]$, Acta Chim. Slov., 2015,62 (2) 378-384.

${ }^{16}$ Hiller W.; Strähle J.; Prinz H.; Dehnicke K.; Die Kristallstruktur von $\mathrm{PPh}_{3} \mathrm{Me}\left[\mathrm{NbOCl}{ }_{4}\left(\mathrm{CH}_{3} \mathrm{CN}\right)\right]$. Zeitschrift für Naturforsch. B 1984, 39 (1), 107-110.

${ }^{17}$ Lide D. R. (editor-in-chief) (2003-2004). "Section 10. Atomic, Molecular, and Optical Physics". Ionization Potentials of Atoms and Atomic Ions. CRC Handbook of Chemistry and Physics (84th edition). CRC Press. pp. 10-178 to 10-180. 